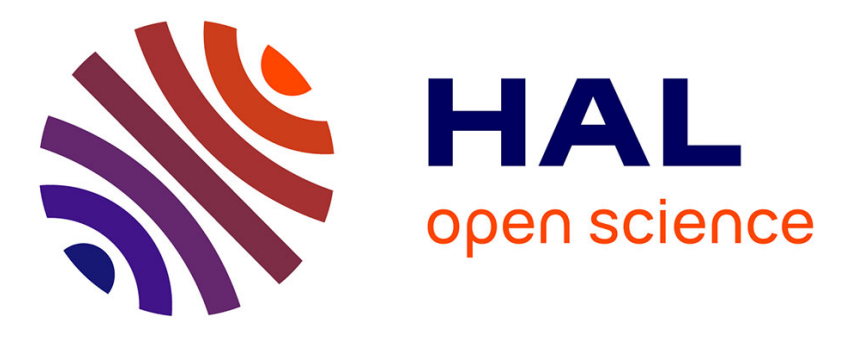

\title{
Sleepiness in sleepwalking and sleep terrors: a higher sleep pressure?
}

Marisol Carrillo-Solano, Smaranda Leu-Semenescu, Jean-Louis Golmard, Elisabeth Groos, Isabelle Arnulf

\section{To cite this version:}

Marisol Carrillo-Solano, Smaranda Leu-Semenescu, Jean-Louis Golmard, Elisabeth Groos, Isabelle Arnulf. Sleepiness in sleepwalking and sleep terrors: a higher sleep pressure?. Sleep Medicine, 2016, 10.1016/j.sleep.2015.11.020 . hal-01286061

\section{HAL Id: hal-01286061 https: / hal.sorbonne-universite.fr/hal-01286061}

Submitted on 10 Mar 2016

HAL is a multi-disciplinary open access archive for the deposit and dissemination of scientific research documents, whether they are published or not. The documents may come from teaching and research institutions in France or abroad, or from public or private research centers.
L'archive ouverte pluridisciplinaire HAL, est destinée au dépôt et à la diffusion de documents scientifiques de niveau recherche, publiés ou non, émanant des établissements d'enseignement et de recherche français ou étrangers, des laboratoires publics ou privés. 


\section{Sleepiness in Sleepwalking and Sleep Terrors: a Higher Sleep Pressure?}

Marisol Carrillo-Solano ${ }^{\mathrm{a}}$, Smaranda Leu-Semenescu ${ }^{\mathrm{a}, \mathrm{c}}$, Jean-Louis Golmard ${ }^{\mathrm{b}}$, Elisabeth Groos $^{\mathrm{a}}$ and Isabelle Arnulf $\mathrm{f}^{\mathrm{a}, \mathrm{c}, \mathrm{d}}$

${ }^{a}$ Sleep Disorders Unit and ${ }^{b}$ Biostatistics Department of the Pitié-Salpêtrière University Hospital, APHP, Paris, France, ${ }^{\mathrm{c}}$ Sorbonne University, UPMC Univ Paris 06, IHU neuroscience, ${ }^{d}$ Brain Research Institute (CRICM), Inserm UMR-S975, CNRS UMR7225, Paris, France

Running head: Sleepiness in sleepwalking

\section{Highlights}

- Half of 158 adult patients with sleepwalking or sleep terrors report excessive daytime sleepiness

- They fall asleep more rapidly (with rapid N3 and REM sleep onsets) and sleep longer than non-sleepy sleepwalkers

- The level of sleepiness is independent of the clinical severity of sleepwalking and of sleep disturbances

- This higher sleep pressure in sleepy sleepwalkers may contribute to incomplete arousals from N3. 


\section{ABSTRACT}

Objective: To identify the determinants of excessive daytime sleepiness in adults with sleepwalking or sleep terrors (SW/ST).

Methods: We collected the charts of all consecutive adult patients admitted from 2012 to 2014 for SW/ST. They had completed the Paris Arousal Disorders Severity Scale and the Epworth sleepiness scale, and had undergone one $(n=34)$ or two consecutive $(n=124)$ nocturnal videopolysomnographies. The demographic, clinical and sleep determinants of excessive daytime sleepiness (defined as an Epworth score greater than 10) were analyzed.

Results: Almost half (46.8\%) of the 158 adult patients with SW/ST reported excessive daytime sleepiness. They had shorter sleep onset latencies (in Night 1 and Night 2), shorter REM sleep latencies, longer total sleep time and higher REM sleep percentages in Night 2, but no greater clinical severity of the parasomnia than patients without sleepiness. The level of sleepiness correlated with the same measures (sleep onset latency on both nights, REM sleep onset latency and total sleep time in Night 2), plus the latency to N3. In the regression model, higher sleepiness was determined by shorter sleep onset latency on Night 1 , lower number of awakenings in N3 on Night 1 and higher total sleep time on Night 2.

Conclusion: Daytime sleepiness in patients with SW/ST is not the consequence of disturbed sleep but is associated with a specific polygraphic phenotype (rapid sleep onset, long sleep time, lower numbers of awakenings on N3), suggestive of a higher sleep pressure that may contribute to incomplete arousal from N3.

Key-words: Parasomnia, Sleepwalking, Sleep terrors, Sleepiness, Sleep pressure 


\section{Introduction}

Sleepwalking (SW) and sleep terrors (ST) are inappropriate, mostly amnestic, behaviors occurring upon a partial arousal from N3 sleep [1]. These non-REM sleep parasomnias are common in children but may persist (and sometimes appear) in up to 2-4\% adults [2, 3]. Sleep terrors commonly overlap with sleepwalking in adults, either at the same moment (e.g., fear behavior and shouting, followed by a frenzied escape from the bed) or at different periods during the night. In addition to causing injuries to self and others, these nocturnal parasomnias impact upon daytime functioning, as recently shown by several clinical works [4]. Adults with SW/ST often feel ashamed and anxious about their disorder, and they report lower quality of life, more difficulties in initiating and maintaining sleep, higher levels of anxiety, and daytime fatigue than healthy controls [5]. Excessive daytime sleepiness is in particular a recent matter of concern in sleepwalking. Indeed, a score greater than 10 on the Epworth sleepiness scale is reported by $47 \%$ of 43 patients with SW/ST in Paris, France [6], $42 \%$ of 100 adult sleepwalkers in Montpellier, France [5], 41\% of 71 patients with SW/ST in Paris, France [7], $46 \%$ of 70 adult sleepwalkers in Montreal, Canada [8], but only $15 \%$ of 32 young subjects with sleepwalking in Prague, Czech Republic [9]. This increased feeling of sleepiness is substantiated by a mean daytime sleep onset latency shorter than 8 min on multiple sleep latencies in 7/10 sleepwalkers in a small series [10], whereas only the first morning nap of 4 naps occurred more rapidly in patients than in controls in a larger series [11].

Why are nearly half of adult sleepwalkers feeling abnormally sleepy during the daytime? Is excessive sleepiness a consequence of fragmented sleep (especially fragmented N3), of nonrestorative sleep properties, or of the clinical severity of the disorder? Recently, some of these questions were examined in 70 patients with SW studied during a single night. No common sleep characteristics, including the intensity of slow wave activity (a marker of sleep 
restorative properties that may be altered in sleepwalking/sleep terrors) or the number of $\mathrm{N} 3$ awakenings, predicted the daytime level of sleepiness [8]. We aimed to replicate this study in a larger sample, taking advantage of retrospective analysis of routine patients charts in our unit, who underwent not one but two consecutive nights of polysomnography (a routine procedure which increases the chance of findings markers of SW/ST, and could allow to avoid first night effects and post-deprivation sleep rebound), and to examine, in addition, whether the characteristics and severity of SW/ST, measured using the Paris Arousal Disorder Severity Scale [7], would determine the level of daytime sleepiness in adults with SW/ST.

\section{Methods}

\subsection{Patients}

All consecutive patients with confirmed SW and ST seen in the adult sleep disorder unit of the Pitie-Salpetriere university hospital were included from January 2012 to June 2014. They had to meet the international criteria for SW and ST, including the following: (1) a history of SW defined by complex behaviors that are usually initiated during arousals from slow-wave sleep and culminating in walking around with an altered state of consciousness and impaired judgment; or (2) a history of ST defined by autonomic nervous system responses of confusional arousal episodes, sitting up in bed, perhaps thrashing about or crying or screaming inconsolably, with a fast heart rate and rapid breathing, and all of these episodes were accompanied by misperceptions of the environment, impaired judgment, and frequent retrograde amnesia as assessed by the patient and bed partner or parents [1]. Patients with parasomnia overlap disorders (wherein sleepwalking/sleep terrors are combined with REM sleep behavior disorders), nocturnal epilepsy, sleep sex or sleep-related eating disorders (as the exclusive symptoms) and patients with psychosis or concomitant severe psychiatric 
disorders (including current depression) were excluded. The clinical diagnosis of ST and SW was, in addition, supported by measures from videopolysomnography, performed to exclude other diagnoses and to document either confusional arousals, frequent (more than 3) awakenings from N3 or dissociated arousals (coexistence of markers of local sleep and local waking, either in the same or in different EEG channels), as a clinical routine in the sleep unit. Because this study was a retrospective collection of clinical and polysomnographical measures from chart review, the local ethics committee (CPP Paris IDF 6) waived written consent from the patients, provided that they had given their oral consent for their data to be anonymously collected, which was the case here.

\subsection{Clinical investigations}

All patients were interviewed by a sleep physician (neurologist or psychiatrist), including the screening for any concomitant anxiety or mood disorder. They routinely completed the Epworth sleepiness scale (ESS) at time of the videopolysomnography [12]. One hundred and eighteen patients (20 having spent one night and 98 having spent two consecutive nights) completed the Paris Arousal Disorders Severity Scale (PADSS) composed of three parts. The PADSS-A is an inventory of 17 abnormal amnestic behaviors scored as never (0), sometimes (1) and often (2) and yields a maximum score of 26; PADSS-B evaluates the frequency of behavioural episodes from 0 (never) to 34 (several times per night); PADSS-C evaluates the general consequences of the disorder, including injury to oneself ( 0 to 2$)$ or others ( 0 to 2$)$, disturbance of others' sleep (0 to 2), morning tiredness (0 to 2), and psychological (shame, anxiety) consequences ( 0 to 2 ), yielding a maximum of 10 [7]. The total of the scale is 50 and a score greater than 13 has a high sensitivity and specificity for separating patients with SW/ST from healthy controls and from patients with REM parasomnias. 


\subsection{Video and sleep monitoring}

The videopolysomnography was performed over two consecutive nights in 124 patients and a single night in 34 additional patients (mostly because they could not stay for a second night or because we could not rapidly offer them a second night). It included 10 electroencephalography (EEG) leads (A1; A2; Fp1; C3; T3; O1; Fp2; C4; T4, O2) acquired in a monopolar referential montage and displayed on screen as bipolar, with sleep stages scored on the Fp1-C3, C3-A2 and C3-O1 channels, two electro-oculograms (EOG) (left inferior epicanthus/right mastoid; right superior epicanthus/right mastoid), three surface electromyographs (EMG) levator menti and right and left tibialis anterior. The patients were recommended to keep regular sleep-wake schedules with no sleep deprivation during the week preceding the sleep monitoring, but they did not complete any sleep log. Almost all of them did not take any psychotropic drugs, and the rare patients on psychotropic drug stopped them for at least 7 drug half-lives before the sleep tests. Patients arrived between 4 PM and 7 PM, were hooked, and had diner in the unit. Sleep was monitored from lights off (ad libitum, but no later than $11 \mathrm{PM}$ ) to lights on (ad libitum) during both nights. Patients stayed in the unit between Night 1 and Night 2, performing quiet activities including answering physicians interviews, examination and questionnaires, reading, phoning, working on their computer, or walking in the hospital garden, but were not allowed to sleep, as checked by the nurses. The slow wave activity was measured on Fp1-C3, which was not conventional but collected EEG activity of amplitude similar to the conventional F3-A2 and was exactly measured in the same way in all patients, whatever their group. Nasal pressure, tracheal sounds, chest and abdomen movements via contention belts, electrocardiogram (EKG), pulse oximeter, position, as well as synchronized ambiance sounds and infrared video. The scoring of the sleep stages was performed by trained neurologists according to standard methods [13]. The analysis of 
videopolysomnography included in addition the number of awakenings from stage N3 sleep over the 2 nights, as well as the corresponding observed behaviors on the video, scored by two scorers. Any abnormal behavior upon awakening in the N3 stage (opening eyes, raising head, exploring the environment with an anxious gaze, raising trunk, standing up, walking, being violent, speaking, shouting) was scored as a confusional behavior. All respiratory events were measured, including apnea, hypopnea, and flow limitation (defined as a plateau on nasal pressure lasting at least $10 \mathrm{sec}$ and followed by an arousal).

\subsection{Statistical analysis}

Data are presented as means $\pm \mathrm{SD}$, unless otherwise specified. The comparisons between the patients with and without daytime sleepiness (defined as an ESS score greater than 10) were performed using Student's t-tests (quantitative measures) and chi-squared tests (qualitative measures). Pearson correlations were performed between quantitative or ordinal measures and the ESS score. All tests were two-sided and a p-value below 0.05 was considered statistically significant. All variables which achieved a p-value less than 0.15 were entered into a stepwise regression model. The computations were performed by the statistician using the SAS-V9 statistical package (SAS Institute Inc, Cary, NC, USA).

\section{Results}

\subsection{Demographic and clinical characteristics of the patients}

There were 158 patients (53.2\% women and $46.8 \%$ men) with SW/ST, aged $32 \pm 10$ years (mean $\pm \mathrm{SD}$ ) at the time of the videopolysomnography, of whom $45 \%$ had frequent sleep terrors (Table 1). Among them, 74 (46.8\%) had an ESS greater than 10, and 26 (16.4\%) 
had an ESS greater than 15. There were no differences in age, sex, presence of sleep terrors, or the global severity of the parasomnia. In terms of behaviors, monthly frequency and consequences (as evaluated with the PADSS) did not differ between the groups with and without excessive daytime sleepiness (Table 1). The exclusion of item C-22 ("I am tired on the morning”) from the PADSS total score and PADSS-C sub-score did not change these negative results.

Table 1

In addition, none of the individual items of the PADSS-A and PADSS-C were different between groups, except for Item A-11 (“I handled or moved heavy objects”), which was scored more frequently in the group with vs. without sleepiness (Table 2). Item 16 ("eating food") tended to be more frequent in the patients with vs. without sleepiness.

\section{Table 2}

\subsection{Sleep measures}

During the first night, the 74 patients with excessive daytime sleepiness had shorter sleep onset latencies, and they tended to have a shorter latency to N3, and to have lower 
numbers of $\mathrm{N} 3$ awakenings than the 84 patients without excessive sleepiness (Table 3). There were no other differences in sleep measures, including markers of sleep continuity (total sleep time, sleep efficiency), architecture (percentages of sleep stages), and fragmentation (presence of any confusional behavior upon awakening from N3 sleep, number of N3 awakenings, arousal index, periodic leg movements or apnea-hypopnea). In the full group, 17 (10.7\%) patients had between 5 and 15 apnea-hypopnea/h, $8(5 \%)$ had more than 15 and less than 30 apnea-hypopnea/h, and $1(0.06 \%)$ patient had an apnea-hypopnea index of 30.5. These percentages did not differ between groups with and without sleepiness. Only 3 patients (including 1 with sleepiness) had a periodic leg movement index greater than 15 .

\section{Table 3}

During the second night (Table 3), completed in 124/158 patients, the 57 patients with excessive daytime sleepiness had shorter sleep onsets and REM sleep latencies, longer total sleep times, higher REM sleep percentages, and a lower difference between total sleep time on Night 2 and Night 1 (a variable that we suggested to be linked to previous sleep debt) than the 67 patients without excessive daytime sleepiness. As expected by first night effects, the latencies to sleep onset, N3 and REM onsets were longer in the first than in the second night, whereas total sleep time as well as N2 and REM sleep percentages were shorter in the first than in the second night. 
The ESS was higher when the sleep onset latency at night was shorter on Night $1(r=$ $-0.19 ; \mathrm{P}=0.02)$ and on Night $2(r=-0.28 ; \mathrm{P}=0.002)$, when the latency from sleep onset to $\mathrm{N} 3$ sleep was shorter on Night $1(r=-0.2 ; \mathrm{P}=0.012)$ and on Night $2(r=-0.2 ; \mathrm{P}=0.03)$, when the latency to REM sleep was shorter on Night $2(r=-0.19 ; \mathrm{P}=0.03)$ but not on Night 1 , and when the total sleep time on Night 2 was higher $(r=0.22 ; \mathrm{P}=0.014)$. The ESS did not significantly correlate with any other clinical variables (age, severity of parasomnia as assessed by each sub-score of the PADSS as well as the total score) or measures of sleep duration (total sleep time during the two nights, sleep efficacy, wakefulness after sleep onset), architecture (percentages and duration of sleep stages), fragmentation (number of arousals/awakenings in N3 sleep, arousal index, apnea-hypopnea index, periodic leg movements index) or an indirect marker of sleep deprivation (difference of N3 between Night 2 and Night 1). Similarly, categorical measures of demography (sex), clinical aspects (score at each descriptive item of PADSS-A, presence of sleep terror) and videopolygraphical aspects (presence of abnormal behavior during N3 awakening, presence of intense adrenergic reaction upon N3 awakening) did not predict any change in ESS.

\section{Table 4}

All measures with a $P<0.15$ were entered into a stepwise regression model, including sleep onset latencies on Nights 1 and 2, N3 latencies on Nights 1 and 2, total sleep time on Night 2, plus sleep efficacy on Night $1(r=0.14 ; P=0.086)$, and the number of awakenings in N3 during Night $1(r=-0.14, \mathrm{P}=0.09)$. The final model is indicated in Table 3 . The level of 
sleepiness was determined by the sleep onset latency on Night 1, the number of awakenings in N3 on Night 1 and the total sleep time on Night 2.

\section{Discussion}

Almost half of the 158 adult patients with sleepwalking and sleep terrors reported excessive daytime sleepiness. They had shorter sleep onset latencies (in Night 1 and Night 2), shorter REM sleep latencies, longer total sleep time and higher REM sleep percentages in Night 2 than patients without sleepiness. Similarly, the level of sleepiness correlated with the same measures (sleep onset latency in both nights, REM sleep onset latency and total sleep time in Night 2), plus the latency to N3. The final regression model indicated that the level of sleepiness was determined by the sleep onset latency on Night 1 , the number of awakenings in N3 on Night 1 and the total sleep time on Night 2.

The high frequency (46.8\%) of patients with excessive daytime sleepiness among adult sleepwalkers found here corroborates recent observations in large groups of sleepwalkers, which have also reported that $41-47 \%$ of patients had ESS greater than 10 [5, 6, 8]. This frequency was systematically higher than the frequency in age-matched healthy controls $[5,6,8]$. Because it exceeds the $10 \%$ of naturally hypersomnolent subjects found in the generally healthy population $[14,15]$, this high frequency could not result from an overlap between natural hypersomnolence and sleepwalking.

Sleepiness in our sleepwalkers group was not linked to age, gender, arousal index, wakefulness after sleep onset, periodic leg movements, or sleep-disordered breathing, as already observed in a smaller group [8]. More surprisingly, it was not associated either with more severe forms of the parasomnia (as measured by the frequency of SW/ST episodes, the complexity of nocturnal behaviors or their association with injuries), or to specific forms 
including some degrees of sleep terrors, sleep-related eating disorder or sexsomnia. Another group even finds that sleepiness is more frequent in sleepwalkers with infrequent than frequent episodes [5]. One may argue that patients with SW/ST have the greatest difficulty in evaluating the frequency of their episodes, because most of them are not recalled, making the correlation between SW/ST frequency and sleepiness less reliable. However, we do not find either a correlation between the number of awakenings in N3 sleep or the presence of confusional behaviors on videopolysomnography (which are more objective makers than selfevaluation of the number of parasomnia episodes, at least during here two nights), and the daytime sleepiness. The number of awakenings in N3 is one of the determinants of the level of sleepiness in the final statistical model, but in an inverse direction than expected (lower number, higher sleepiness). An exception is the behavior of handling heavy objects, which is more frequently reported by sleepy than non-sleepy sleepwalkers (with some difficulties in interpreting this finding) and a trend for sleepy patients to display more frequently sleepeating related behaviors. In our preceding study, we focused on 15 patients with sleep-eating related disorders; these patients were more sleepy than healthy controls and tended to be more sleepy than sleepwalkers [16]. However, these small differences between sleepy and nonsleepy sleepwalkers regarding amnestic handling and eating behaviors disappear in the general model, suggesting that they are dependent upon other sleep characteristics.

Several sleep measures were different here between the sleepy and non-sleepy groups, contrary to the preceding study performed in 70 French Canadian patients, which found no polygraphical markers associated with daytime sleepiness (even using spectral analysis, which was not performed here), but used a smaller sample [8]. These measures included shorter sleep onset latencies (a link repeated during the two nights), a shorter latency to REM sleep, a longer total sleep time and a higher REM sleep percentage in Night 2. If none of these measures are linked to the N3 sleep (the stage from which the parasomnia arises), they 
coherently indicate a propensity to fall asleep (more rapid sleep and REM sleep onsets) and to show prolonged sleep (as shown by longer sleep time, which is usually associated with longer REM sleep). Longer sleep time in sleepy patients during Night 2 does not result from previous sleep deprivation (a strong trigger of sleepwalking episodes) [17], because it is observed on the second and not on the first night, and because there are no differences between sleep times on Night 1 and Night 2, in contrast to non-sleepy patients who exhibited a longer sleep time on Night 1 than on Night 2. All in all, these results suggest that sleepiness in sleepwalking is not caused by sleep deprivation or fragmented sleep (via arousals, upper airway resistance, or N3 awakenings), but is, on the contrary associated with a phenotype of “good sleeper" falling rapidly asleep, and sleeping for a long time. This phenotype suggests a higher sleep propensity in sleepwalkers with than without sleepiness (close to the hypersomnolence of central origin), which could be a genuine subtype of sleepwalking. One may even imagine that this higher sleep pressure and subsequent sleepiness is not a consequence but one of the causal mechanisms of SW/ST in vulnerable subjects. Indeed, subjects with higher sleep pressure would have more difficulties waking from N3, possibly resulting in a partial rather than a complete arousal from N3, leading to this hybrid state of half-wake/half-sleep which characterizes SW and ST [18]. Such hypotheses cannot explain why these people awake from N3, but rather how they awake. As we did not perform a spectral EEG analysis, it is not possible to evaluate whether the homeostatic sleep drive is intact or increased in sleepy vs. non-sleepy sleepwalkers. The heritability of sleepwalking is high, especially in adults [19], and a genetic locus for sleepwalking has been found at chromosome 20q12- q13.12 in a multiplex family [20], suggesting that allelic variants may underlie the presence of sleepwalking and sleep terrors. Once these variants will be found, it will be interesting to study whether they are different in sleepy vs. non-sleepy patients. 
Conversely, it will be also interesting to observe whether the "sleepy" phenotype of SW/ST has a familial aggregation too.

This study contains several limitations inherent to any retrospective collections of clinical and polygraphical measures. Only $75 \%$ of patients completed the PADSS, but their number $(\mathrm{n}=118)$ was sufficient to provide robust analysis of correlation with ESS. Similarly, $78 \%$ of patients underwent two consecutive nights, but their number $(n=124)$ was again sufficient to provide robust analysis. There were no specific questionnaires evaluating insomnia, depression (a frequent cause of sleepiness) or anxiety, but anxiety and mood disorders were screened by the physician in charge, and the answers on Item 23 of the PADSS-C (which evaluates the degree of shame, anxiety, fear to go to sleep or any psychological association with the parasomnia) were similar between patients with and without sleepiness. The level of sleep deprivation was not measured using sleep log or (even better) actigraphy during the week preceding the videopolysomnographies. Patients were however recommended to keep regular sleep-wake schedule before the test, and we used a surrogate measure of sleep deprivation, which indicated no sleep deprivation in the sleepy patients, in contrast to the non-sleepy patients. Eventually, sleepiness was measured using the ESS, and not with objective measures of sleepiness or alertness (including multiple sleep latency test, which may not be enough sensitive to capture the sleepiness problems in patients with SW/ST [11], maintenance of wakefulness tests, attention tests). All these measures have their own interest and do not measure exactly the same thing [21]. The Epworth sleepiness scale remains a robust measure of self-perceived sleepiness, which is what disturbs the patient.

In conclusion, sleepiness in SW/ST is not the consequence of disturbed sleep but is, on the contrary, associated with a specific polygraphic phenotype (rapid sleep onset, long sleep time, lower awakenings in N3), suggestive of a higher sleep propensity, which may contribute 
to the imperfect awakenings from N3 characteristic of SW/ST. This frequent subtype of sleepwalking would be worth studying in terms of genetics.

\section{Author contribution}

The patients were diagnosed (clinical diagnosis and scoring) and followed up by IA, SLS and EG. The measures were collected by MCS, who prepared the tables and figures. JLG performed the statistics. IA wrote the article, which was reviewed and approved by all authors.

\section{Financial Disclosure Statement}

This is not an industry-funded study. It was performed as part of the normal work of the participants.

\section{Conflict of interest}

The authors have no conflict of interest related to the study. Professor Arnulf received an honorarium from UCB Pharma for speaking engagements and consultancy. Dr. LeuSemenescu had her congress fees paid by UCB Pharma. These financial disclosures are unrelated to the present study and topic. Drs. Carrillo-Solano and Golmard have no conflicts of interest to disclose.

\section{References}

[1]. American Academy of Sleep Medicine. The international Classification of Sleep Disorders, 3rd edition. Darien, IL: American Academy of Sleep Medicine; 2014. 
[2]. Ohayon MM, Guilleminault C, Priest RG. Night terrors, sleepwalking, and confusional arousals in the general population: their frequency and relationship to other sleep and mental disorders. J Clin Psychiatry 1999; 60: 268-76; quiz 77.

[3]. Ohayon MM, Mahowald MW, Dauvilliers Y, et al. Prevalence and comorbidity of nocturnal wandering in the U.S. adult general population. Neurology 2012; 78: 1583-9.

[4]. Zadra A, Desautels A, Petit D, et al. Somnambulism: clinical aspects and pathophysiological hypotheses. Lancet Neurol 2013; 12: 285-94.

[5]. Lopez R, Jaussent I, Scholz S, et al. Functional impairment in adult sleepwalkers: a case-control study. Sleep 2013; 36: 345-51.

[6]. Oudiette D, Leu S, Pottier M, et al. Dreamlike mentations during sleepwalking and sleep terrors in adults. Sleep 2009; 32: 1621-7.

[7]. Arnulf I, Zhang B, Uguccioni G, et al. A scale for assessing the severity of arousal disorders. Sleep 2014; 37: 127-36.

[8]. Desautels A, Zadra A, Labelle M, et al. Daytime somnolence in adult sleepwalkers. Sleep Med 2013; 14: 1187-91.

[9]. Bušková J, Piško J, Pastorekb L, et al. The course and character of sleepwalking in adulthood: a clinical and polysomnographic study. Behav Sleep Med 2015; 13: 169-77.

[10]. Montplaisir J, Petit D, Pilon M, et al. Does sleepwalking impair daytime vigilance? . J Clin Sleep Med 2011; 7: 219.

[11]. Lopez R, Jaussent I, Dauvilliers Y. Objective daytime sleepiness in patients with somnambulism or sleep terrors. Neurology 2014; 83: 2070-6.

[12]. Johns MH. A new method for measuring daytime sleepiness: the Epworth Sleepiness Scale. Sleep 1991; 14: 540-5. 
[13]. Iber C, Ancoli-Israel S, Chesson A, et al. The AASM Manual for the Scoring of Sleep and Associated Events: Rules, Terminology and Technical Specifications, Irst Ed. Medecine AAoS, editor. Westchester, IL: American Academy of Sleep Medecine; 2007.

[14]. Johns M, Hocking B. Daytime sleepiness and sleep habits of Australian workers. Sleep 1997; 20: 844-9.

[15]. Parkes JD, Chen SY, Clift SJ, et al. The clinical diagnosis of the narcoleptic syndrome. J Sleep Res 1998; 7: 41-52.

[16]. Brion A, Flamand M, Oudiette D, et al. Sleep-related eating disorder versus sleepwalking: a controlled study. Sleep Med 2012; 13: 1094-101.

[17]. Zadra A, Pilon M, Montplaisir J. Polysomnographic diagnosis of sleepwalking: effects of sleep deprivation. Ann Neurol 2008; 63: 513-9.

[18]. Terzaghi M, Sartori I, Tassi L, et al. Evidence of dissociated arousal states during NREM parasomnia from an intracerebral neurophysiological study. Sleep 2009; 32: 409-12. [19]. Hublin C, Kaprio J, Partinen M, et al. Prevalence and genetics of sleepwalking: a population-based twin study. Neurology 1997; 48: 177-81.

[20]. Licis A, Desruisseau D, Yamada K, et al. Novel genetic findings in an extended family pedigree with sleepwalking. Neurology 2011; 76: 49-52.

[21]. Chervin RD. Epworth sleepiness scale? . Sleep Med 2003; 4: 175-6. 
Table 1

Clinical characteristics of patients with sleepwalking and sleep terrors, with and without excessive daytime sleepiness

\begin{tabular}{|c|c|c|c|c|}
\hline & All patients & $\begin{array}{c}\text { With } \\
\text { sleepiness }\end{array}$ & $\begin{array}{l}\text { Without } \\
\text { sleepiness }\end{array}$ & $\mathrm{P}$ \\
\hline $\mathrm{N}$ & 158 & 74 & 84 & \\
\hline Age, y & $32.8 \pm 9.9$ & $33.6 \pm 9.8$ & $32.2 \pm 10.1$ & 0.37 \\
\hline Gender, $\%$ men & 47.6 & 43.2 & 50 & 0.43 \\
\hline ESS score, 0-24 & $9.8 \pm 5.4$ & $14.3 \pm 3.1$ & $5.8 \pm 3.1$ & $<0.0001$ \\
\hline \multicolumn{5}{|c|}{ Clinical severity of the parasomnia } \\
\hline $\mathrm{N}$ & 118 & 48 & 62 & \\
\hline Sleep terrors, \% & 45 & 46.9 & 43.4 & 0.73 \\
\hline Total PADSS, 0-50 & $17.4 \pm 5.3$ & $18.1 \pm 4.5$ & $16.9 \pm 5.8$ & 0.26 \\
\hline PADSS-A, 0-34 & $9.0 \pm 5.0$ & $9.8 \pm 5.2$ & $8.3 \pm 4.7$ & 0.09 \\
\hline PADSS-B, 0-6 & $4.2 \pm 1.1$ & $4.3 \pm 1.0$ & $4.1 \pm 1.1$ & 0.32 \\
\hline Number of & $17.4 \pm 21.8$ & $20.1 \pm 22.6$ & $14.8 \pm 20.7$ & 0.21 \\
\hline \multicolumn{5}{|l|}{ episodes/month } \\
\hline PADSS-C, 0-10 & $3.7 \pm 1.5$ & $3.8 \pm 1.4$ & $3.7 \pm 1.7$ & 0.59 \\
\hline
\end{tabular}

ESS: Epworth sleepiness scale; PADSS: Paris arousal disorder sleepiness scale. 


\section{Table 2}

Behaviors during sleepwalking and sleep terrors in patients with and without excessive daytime sleepiness

\begin{tabular}{|c|c|c|c|}
\hline PADSS-A, \% patients with positive answer & $\begin{array}{c}\text { With } \\
\text { sleepiness }\end{array}$ & $\begin{array}{l}\text { Without } \\
\text { sleepiness }\end{array}$ & $\mathrm{P}$ value \\
\hline I screamed & 93.5 & 84.6 & 0.10 \\
\hline I sat up in my bed & 95.7 & 87.7 & 0.48 \\
\hline I hit or kicked someone or something, & 72.3 & 64.1 & 0.50 \\
\hline I fell out of bed & 38.3 & 41.3 & 0.76 \\
\hline I went out of my room & 72.3 & 69.2 & 0.40 \\
\hline I went down or up the stairs & 34.1 & 30.2 & 0.87 \\
\hline I went out of my home & 14.9 & 17.2 & 0.83 \\
\hline I opened a window & 42.6 & 30.8 & 0.23 \\
\hline I climbed out a window & 12.8 & 10.9 & 0.57 \\
\hline $\begin{array}{l}\text { I handled or moved light objects (slippers, small } \\
\text { things) }\end{array}$ & 61.7 & 58.5 & 0.26 \\
\hline $\begin{array}{l}\text { I handled or moved heavy objects (lamp, vase, } \\
\text { furniture) }\end{array}$ & 44.7 & 28.1 & 0.02 \\
\hline I broke an object, window, or wall & 23.4 & 30.8 & 0.48 \\
\hline I picked up sharp objects (knife, tools) & 6.3 & 6.4 & 0.97 \\
\hline $\begin{array}{l}\text { I manipulated objects that may set a fire (matches, } \\
\text { lighter, gas stove, oven) }\end{array}$ & 2.1 & 1.6 & 0.82 \\
\hline $\begin{array}{l}\text { I touched things around windows and openings } \\
\text { (blinds, shutters, curtains, window/door handle) }\end{array}$ & 48.9 & 47.7 & 0.49 \\
\hline
\end{tabular}


I prepared or ate some food or a drink

I unwillingly performed a sexual act

PADSS-C

I disturb someone else's sleep

I injured myself

I hurt someone

I am tired the next day

It disturbs me psychologically (feeling of shame, anxiety, fear to go to bed, ...)
25.5

$13.9 \quad 0.08$

12.8

10.8

0.42

89.4

93.5

0.63

38.3

43

0.61

21.3

23.1

0.82

91.5

81.5

0.14

72.3

70.8

0.86

P: significance of the chi-square test comparing the percentage of sleepy vs. not sleepy patients. 
Table 3

Sleep characteristics in patients with sleepwalking/sleep terrors, with and without excessive daytime sleepiness

\begin{tabular}{|c|c|c|c|c|}
\hline & All patients & $\begin{array}{c}\text { With } \\
\text { sleepiness }\end{array}$ & $\begin{array}{l}\text { Without } \\
\text { sleepiness }\end{array}$ & $\mathrm{P}$ \\
\hline Night $1, \mathrm{n}$ & 158 & 74 & 84 & \\
\hline \multicolumn{5}{|l|}{ Latency to, min } \\
\hline Sleep onset & $33.8 \pm 31.8$ & $26 \pm 21.1$ & $41.1 \pm 37.8$ & 0.002 \\
\hline N3 sleep & $53.3 \pm 38.5$ & $46.8 \pm 36.8$ & $58.5 \pm 39.7$ & 0.06 \\
\hline REM sleep & $140.5 \pm 78.7$ & $142.3 \pm 80.4$ & $134.3 \pm 74.5$ & 0.47 \\
\hline Total sleep time, $\min$ & $469.1 \pm 93.6$ & $461.5 \pm 96.5$ & $477.5 \pm 92$ & 0.29 \\
\hline Sleep efficiency, $\%$ & $86.5 \pm 8.5$ & $87.6 \pm 8.2$ & $85.5 \pm 8.8$ & 0.13 \\
\hline \multicolumn{5}{|l|}{ Duration of stages (\% of } \\
\hline \multicolumn{5}{|l|}{ total sleep time) } \\
\hline N1 & $4.3 \pm 3.5$ & $4.2 \pm 3.4$ & $4.4 \pm 3.6$ & 0.73 \\
\hline $\mathrm{N} 2$ & $51.1 \pm 8.7$ & $51.6 \pm 8.2$ & $50.3 \pm 8.8$ & 0.35 \\
\hline $\mathrm{N} 3$ & $25.2 \pm 9.1$ & $25.4 \pm 9.3$ & $25.2 \pm 9.1$ & 0.90 \\
\hline REM & $19.5 \pm 7.5$ & $18.7 \pm 5.7$ & $20.5 \pm 8.7$ & 0.13 \\
\hline \multicolumn{5}{|l|}{ Awakenings from N3 } \\
\hline Number & $5.4 \pm 3.4$ & $5.0 \pm 3.0$ & $5.9 \pm 3.7$ & 0.09 \\
\hline Confusional behavior, & 82.3 & 81.8 & 82.7 & 0.64 \\
\hline \multicolumn{5}{|l|}{$\%$ patients with } \\
\hline Intense adrenergic & 69.1 & 69 & 69.1 & 1.00 \\
\hline
\end{tabular}


Sleep fragmentation

Arousals, n/h

$11.4 \pm 6.5$

$11.1 \pm 6.9$

$11.8 \pm 6.2$

0.51

Periodic leg

$1.7 \pm 6$

$1.2 \pm 2.9$

$1.4 \pm 5.3$

0.80

movements, $\mathrm{n} / \mathrm{h}$

Apnea-hypopnea, n/h

$4 \pm 8$

$4.3 \pm 9.5$

$3.6 \pm 6.4$

0.61

Night 2, n patients

124

57

67

Latency to, min

Sleep onset

N3 sleep

REM sleep

Total sleep time, min

Sleep efficiency, \%

$40.2 \pm 39.7$

$64.0 \pm 51.7$

$90.6 \pm 43.1$

$455.3 \pm 82$

$87.3 \pm 9.2$

Duration of stages (\% of total sleep time)

N1

N2

N3

REM
$4.8 \pm 5.7$

$46.6 \pm 9.3$

$26.8 \pm 9.7$

$22.1 \pm 5.8$
$31.2 \pm 31.3$

$57 \pm 51.4$

$79.8 \pm 44.7$

$476.2 \pm 79.7$

$87.8 \pm 9$

$86.9 \pm 9.5$

0.58

Sleep fragmentation
N3 awakenings, $n$
$5.7 \pm 3.8$
$5.4 \pm 3.3$
$6.2 \pm 4.1$
0.27
Arousal index, $\mathrm{n} / \mathrm{h}$
$10.0 \pm 5.2$
$9.5 \pm 5.8$
$10.6 \pm 4.6$
0.25
Estimated sleep debt*,
$-22 \pm 108$
$6.3 \pm 112.5$
$-46.7 \pm 99$
0.007
$\min$

*Estimated via the difference of N3 sleep between Night 1 and Night 2, assuming that highest values correspond to the compensation of a more important previous debt. 
Table 4 - Model predicting the presence of sleepiness in patients with sleepwalking and sleep terrors

\begin{tabular}{lcccccc}
\hline Effect & DF & Estimat & Standard & Wald Chi & P & Point \\
& & e & Error & Square & & Estimat \\
& & & & & & e \\
\hline Intercept & 1 & -1.254 & 1.27 & 0.97 & 0.33 & \\
Sleep onset latency (Night & 1 & -0.199 & 0.09 & 4.99 & 0.03 & 0.82 \\
1) & & & & & & \\
Number of awakenings in & 1 & -0.126 & 0.06 & 4.43 & 0.04 & 0.88 \\
N3 (Night 1) & & & & & & \\
Total sleep time (Night 2) & 1 & 0.529 & 0.255 & 4.31 & 0.04 & 1.70 \\
\end{tabular}

